\title{
Corticosteroid-binding globulin is a biomarker of inflammation onset and severity in female rats
}

\author{
Lesley A Hill1,2, Tamara S Bodnar1, Joanne Weinberg ${ }^{1}$ and Geoffrey L Hammond1,2 \\ 1Department of Cellular and Physiological Sciences, University of British Columbia, Vancouver, \\ British Columbia, Canada \\ 2Department of Obstetrics and Gynaecology, University of British Columbia, Vancouver, \\ British Columbia, Canada
}

Correspondence

should be addressed

to G L Hammond

Email

Geoffrey.hammond@ubc.ca

\begin{abstract}
Plasma corticosteroid-binding globulin (CBG) plays a critical role in regulating glucocorticoid bioavailability and is an acute phase 'negative' protein during inflammation. In an adjuvant-induced arthritis model, plasma CBG levels decrease in rats that develop severe inflammation, and we have now determined when and how these reductions in CBG occur. After administering complete Freund's adjuvant or saline intra-dermally at the tail base, blood samples were taken periodically for 16 days. In adjuvant-treated rats, decreases in plasma CBG levels matched the severity of inflammation, and decreases were observed 4 days before any clinical signs of inflammation. Decreases in CBG levels coincided with an $\sim 5 \mathrm{kDa}$ reduction in its apparent size, consistent with proteolytic cleavage, and cleaved CBG lacked steroid-binding activity. At the termination of the experimental period, hepatic Cbg mRNA levels were decreased in rats with severe inflammation. While plasma TNF- $\alpha$ increased in all adjuvant-treated rats, increases in II-4, IL-6, IL-10, IL-13 and IFN- $\gamma$ were only observed in rats with cleaved CBG. Rats with cleaved CBG also exhibited increased spleen weights, and strong negative correlations were observed among CBG, IL-6 and spleen weights, respectively. However, there were no differences in hepatic Cbg mRNA levels in relation to the apparent proteolysis of CBG, suggesting that CBG cleavage occurs before changes in hepatic Cbg expression. Our results indicate that the levels and integrity of plasma CBG are biomarkers of the onset and severity of inflammation. Dynamic changes in the levels and function of CBG likely modulate the tissue availability of corticosterone during inflammation.
\end{abstract}

\author{
Key Words \\ - corticosterone \\ - cytokines \\ - spleen weight \\ - proteolysis \\ - serine protease inhibitor
}

Journal of Endocrinology (2016) 230, 215-225

\section{Introduction}

Produced primarily by the liver, corticosteroid-binding globulin (CBG) is a plasma glycoprotein that binds $\sim 90 \%$ of circulating glucocorticoids, and regulates their bioavailability in target tissues (Lin et al. 2010). Plasma CBG shares structural similarities with clade A serine protease inhibitor (SERPINA) family members (Lin et al. 2010).
However, CBG is not a protease inhibitor, but rather serves as a reservoir for glucocorticoids that are released when its reactive centre loop (RCL) is cleaved by proteases, including neutrophil elastase (Hammond et al. 1990), chymotrypsin (Lewis \& Elder 2014) or the bacterial proteinase, LasB (Simard et al. 2014). 
During inflammation, CBG acts as an acute phase 'negative' protein, and reductions in its plasma levels can be attributed to both proteolytic cleavage (Hammond et al. 1990) and downregulation of its production by the liver (Smith \& Hammond 1992, Emptoz-Bonneton et al. 1997, Bernier et al. 1998). In humans, proteolysis of the RCL of CBG by neutrophil elastase appears to be an early event during inflammatory reactions, rendering CBG nonfunctional and promoting the localized release of CBGbound glucocorticoids at sites of inflammation (Hammond 1990, Perogamvros et al. 2012). Enhanced exposure of tissues to anti-inflammatory glucocorticoids represses cytokine production and activity (Brattsand \& Linden 1996), thereby limiting cytokine-mediated tissue damage (Simon 2003). At the same time, plasma glucocorticoid concentrations increase acutely as a result of hypothalamicpituitary-adrenal axis activation in response to stress (Sapolsky et al. 2000), and likely act in synergy with elevations in inflammatory cytokines, such as interleukin-6 (IL-6), to further reduce plasma CBG production, further amplifying free glucocorticoid exposures (Bartalena et al. 1993, Emptoz-Bonneton et al. 1997, Bernier et al. 1998). During recovery from inflammation, normalization of CBG levels likely plays a role in determining when, and to what extent, glucocorticoids act to restore the normal homeostatic balance.

Several studies in CBG-deficient animals support the idea that CBG plays a vital role during the inflammatory process. For instance, a genetic study revealed that C57BL/6 mice are more sensitive to an acute challenge with TNF- $\alpha$ than DBA/2 mice, and this trait was mapped to the Cbg (SerpinA6) locus (Libert et al. 1999). Recently, we have found that Harlan Sprague-Dawley (SD) rats are deficient in plasma CBG (50\% lower levels), when compared with Charles River SD rats (Bodnar et al. 2015). Harlan SD rats are also more sensitive to inflammatory challenges than Charles River SD rats in an adjuvantinduced arthritis model (Bodnar et al. 2015) or after treatment with lipopolysaccharide (Turnbull \& Rivier 1999). A key role for CBG in these differential responses is further supported by studies in $\mathrm{Cbg}^{-/-}$mice in which survival rates after an acute inflammatory challenge are compromised (Petersen et al. 2006).

Low plasma cortisol levels in patients have led to the identification of several individuals with CBG deficiencies (Gagliardi et al. 2010). Genome-wide sequencing of human populations has also identified numerous other single-nucleotide polymorphisms that cause decreased CBG production or defects in steroid binding, some of which are enriched in specific ethnic groups
(Simard et al. 2015). Although patients with CBG deficiencies have been reported to suffer from a variety of symptoms including chronic pain, fatigue, depression, hypotension and excess body weight (Gagliardi et al. 2010), it remains to be determined how well they cope with severe, acute inflammation. To explore this, we have determined when changes in plasma CBG levels occur during the course of acute inflammation in rats and how this relates to the severity of the inflammatory response.

\section{Materials and methods}

\section{Animals and treatments}

Female Sprague-Dawley rats $(n=24,51-52$ days old) were received from Charles River Laboratories International, Inc. (St. Constant, Canada), and maintained as described previously (Bodnar et al. 2015). Following an acclimatization period of 5 days, pre-treatment (baseline) blood samples were taken from the tail vein, under light isofluorane anaesthesia, between 11:00 and 13:00 h for the preparation of serum, which was stored at $-80^{\circ} \mathrm{C}$ until analysed. Three days later, rats were anaesthetized with isoflurane, and injected with $0.6 \mathrm{mg}$ complete Freund's adjuvant $(n=20)$ prepared as described previously (Zhang et al. 2012) or with physiological saline (control; $n=4)$ ). Rats were then housed with CareFRESH (Healthy Pet, Ferndale, USA) bedding to minimize discomfort, and monitored for signs of pain, discomfort or infection, and for general signs of health including activity, coat quality and ability to rear.

Post-treatment, rats were split into two groups $(n=12$ per group; 2 controls and 10 adjuvant-treated rats). Blood samples $(\sim 50 \mu \mathrm{L})$ from the two groups were alternatively collected between 11:00 and 13:00 h under light isoflurane anaesthesia from the tail vein over a 16-day experimental period. Serum was prepared and stored at $-80^{\circ} \mathrm{C}$ until assayed. At the time of blood sampling, rats were weighed and clinical signs of inflammation were scored and recorded. To calculate clinical scores, each of the four paws was given a score of $0-4$, where $0=$ no signs of inflammation, $1=$ single focus of redness or swelling, $2=$ two or more foci of redness or swelling, $3=$ confluent but not global swelling, $4=$ severe global swelling (Zhang et al. 2012). Rats achieving an overall clinical score $\geq 8$ (out of a possible 16) at any point during the study were classified as developing severe inflammation, whereas rats with a clinical score $<8$ were classified as developing mild-moderate inflammation. One animal with severe inflammation was killed on experimental day 14 for humane reasons, while all other animals were killed

Published by Bioscientifica Ltd. 
on experimental day 16. On the day of termination (08:00-10:00 h), rats were removed from the colony room and decapitated $(<1 \mathrm{~min})$. Trunk blood was collected in tubes containing EDTA, and plasma was stored at $-80^{\circ} \mathrm{C}$ until analysed. In addition, livers and spleens were removed, flash frozen and stored at $-80^{\circ} \mathrm{C}$.

The animal experiments were performed in accordance with the National Institutes of Health Guide for the Care and Use of Laboratory Animals, and approved by the University of British Columbia Animal Care Committee.

\section{Plasma CBG analysis}

Plasma corticosterone-binding capacity of CBG was measured using an established ligand-saturation assay (Hammond \& Lahteenmaki 1983). Briefly, samples were diluted (1:1000-1:3000) in phosphate-buffered saline and stripped of endogenous steroids by incubation with dextran-coated charcoal (DCC) for $30 \mathrm{~min}$ at room temperature followed by centrifugation. Samples were then incubated with $\sim 10 \mathrm{nmol} \mathrm{L}^{-1}\left[{ }^{3} \mathrm{H}\right]$-corticosterone (PerkinElmer Life Sciences) in the absence or presence of excess corticosterone to monitor non-specific binding. After separation of free $\left[{ }^{3} \mathrm{H}\right]$-corticosterone by adsorption with DCC for $10 \mathrm{~min}$ and centrifugation at $0^{\circ} \mathrm{C}$, CBGbound $\left[{ }^{3} \mathrm{H}\right]$-corticosterone in the supernatants was determined in a scintillation spectrophotometer.

To assess CBG integrity, diluted (1:200) plasma samples were subjected to SDS-PAGE and transferred to PVDF membranes using a Trans-Blot turbo transfer system (BioRad). Blots were blocked with 5\% milk-PBST for $2 \mathrm{~h}$ at room temperature and incubated overnight at $4^{\circ} \mathrm{C}$ with polyclonal rabbit anti-mouse CBG antiserum diluted 1:4000 in the 5\% milk-PBST (Scrocchi et al. 1993), followed by a horseradish peroxidase-labelled goat antirabbit IgG antibody (1:10,000; Sigma-Aldrich) for $1 \mathrm{~h}$ at room temperature. Immunoreactive $\mathrm{CBG}$ was then detected with ECL reagent using an ImageQuant LAS4000 (GE Health Care). Total protein concentrations in plasma samples were measured using a Micro BCAProtein Assay Kit (Thermo Fisher Scientific).

\section{Separation and characterization of functional and non-functional CBG in plasma samples}

To characterize CBG in plasma samples from rats with severe inflammation, plasma from two rats were pooled, treated with DCC to remove endogenous steroids and then applied to an 11 $\beta$-hydroxy-andros-4-en-3-oxo-17 $\beta$ - carboxylic acid (HACA)-Sepharose affinity column (Seralini et al. 1989). After washing the column with $100 \mathrm{mmol} \mathrm{L}^{-1}$ Tris-NaCl, steroid-bound CBG was eluted with $1 \mu \mathrm{mol} \mathrm{L}-1$ cortisol in the same buffer. The flow-through, wash and eluent fractions were analysed by corticosterone-binding capacity assay and Western blotting, as described above.

\section{Cbg mRNA measurements}

Total RNA was extracted from liver using Trizol reagent (Thermo Fisher Scientific), followed by purification using an RNeasy kit (Qiagen), as per the manufacturer's instructions. To obtain cDNA, $1 \mu \mathrm{g}$ RNA was reversed transcribed using High-Capacity cDNA Reverse Transcription Kits (Thermo Fisher Scientific). Real-time quantitative PCR (qRT-PCR) was completed using a pre-validated rat Serpina6 ( $\mathrm{Cbg})$ PrimeTime Std qPCR Assay (Integrated DNA Technologies (IDT), Coralville, USA: Assay ID\# Rn.PT.58.02619945) and 5 ng cDNA per reaction. The qRT-PCR reactions were run in duplicate on the Applied Biosystems 7500 Fast Real-Time PCR system (Thermo Fisher Scientific). Hepatic Cbg mRNA levels were normalized to those of rat Gapdh (IDT: Assay ID\# Rn.PT.58.35727291) using the delta-delta Ct method.

\section{Multiplex cytokine immunoassays}

Plasma cytokines (IL-4, IL-5, IL-6, IL-10, IL-13, IFN- $\gamma$ and TNF- $\alpha$ ) were measured in plasma samples collected at termination using the Proinflammatory Panel 2 (rat) V-PLEX kit from Meso Scale Discovery (MSD, Rockville, MD, USA; catalog \#: K15059D-1). The cytokine assays were performed according to the established MSD protocol. The assay plate was read using a MESO QuickPlex SQ 120 and data were analysed using the MSD Discovery Workbench software v. 4.0. Lower limits of detection for cytokines $(\mathrm{pg} / \mathrm{mL})$ are as follows: IL-4 (0.16), IL-5 (6.89), IL-6 (7.18), IL-10 (6.18), IL-13 (0.45), TNF- $\alpha$ (1.04), IFN- $\gamma$ (1.48).

\section{Statistical analyses}

Data (expressed as mean \pm S.D. or S.E.M., as indicated) were analysed using t-tests or analysis of variance (ANOVA), with repeated measures as required, followed by Fisher or Tukey's post hoc tests to examine significant main effects. Differences were considered significant at $P \leq 0.05$, and trends $(0.06>P>0.05)$ were examined, as appropriate. $t$-test, ANOVA and post hoc $\mathrm{p}$ values are shown in results; ANOVA F statistics are included in the figure legends.

Published by Bioscientifica Ltd 


\section{Results}

\section{Body weights and clinical scores}

The body weights of all animals increased over the course of the experiment (i.e. $242.0 \pm 8.7 \mathrm{~g}$ to $285.0 \pm 10.4 \mathrm{~g}$ ), but there were no differences in body weight between groups. Five of the 20 adjuvant-treated animals (25\%) developed inflammation, as indicated by their clinical scores (Fig. 1 in parenthesis), which generally increased over time. The mean time of inflammation onset (i.e. the first day of a clinical score $>0$ ) was $12.8 \pm 0.8$ days.

\section{Time course of changes in plasma CBG levels}

Adjuvant-treated rats had a $30-50 \%$ reduction in plasma CBG levels 24-h post-injection, irrespective of whether or not they developed inflammation, and this decrease resolved by day 3 post-injection (Fig. 1). This was not seen in the saline-treated control rats. When compared with

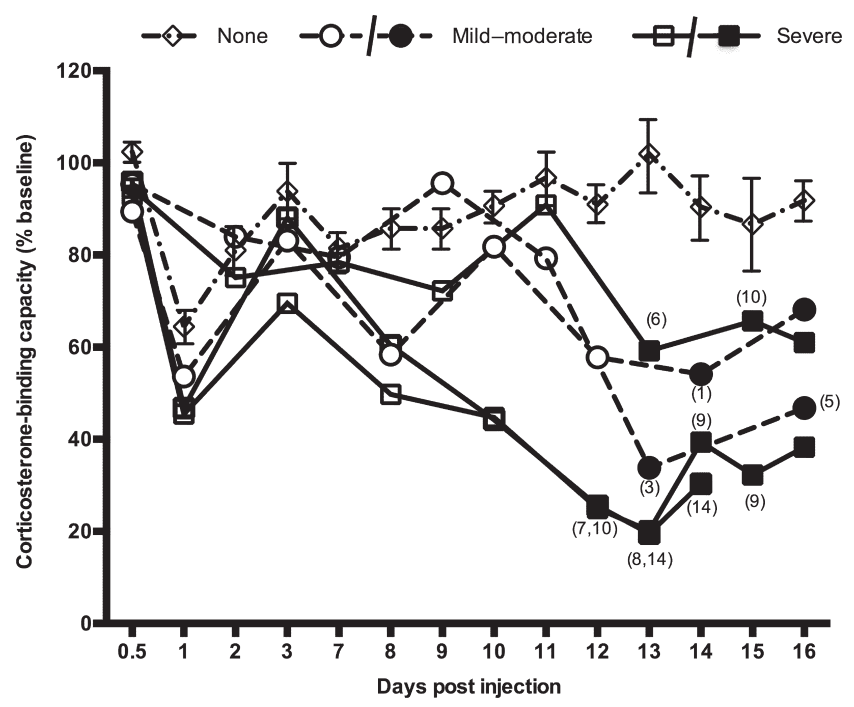

Figure 1

Time course of plasma CBG levels during inflammatory responses. Blood samples were collected from each animal before treatment (baseline values) and after complete Freund's adjuvant injection, as indicated. A reduction in the corticosterone-binding capacity of serum CBG was observed 24-h post-injection in all adjuvant-treated animals. Following this, rats that developed inflammation had large decreases in CBG levels, whereas the mean CBG levels in animals without clinical signs of inflammation (none, diamonds) were unchanged. Rats with mildmoderate inflammation (circles) displayed reduced CBG levels 12-13 days post-injection. Two of three rats with severe inflammation (squares) had reduced CBG levels as early as day 8 , several days before clinical signs of inflammation were evident. Solid symbols denote the presence of clinical symptoms with the corresponding clinical score given in parentheses, while open symbols denote the absence of clinical signs of inflammation. Data for rats $(n=15)$ without inflammation are presented as mean \pm S.E.M. for comparison. baseline values, 40-80\% decreases in CBG-corticosteronebinding capacity were subsequently observed over the experimental period in rats that developed inflammation (Fig. 1), but mean plasma CBG levels were unchanged in saline-treated rats (not shown) or in adjuvant-treated rats that did not develop inflammation (Fig. 1).

Overall, the magnitude of decline in plasma CBG levels matched the severity of inflammation, with animals that developed severe inflammation showing the largest decreases in CBG levels (Fig. 1). In two severely inflamed rats, 50\% reductions in plasma CBG were seen as early as day 8 post-injection, which was 4 days before any clinical signs of inflammation were evident. In these animals, CBG levels reached as low as 20\% of baseline by 12-13 days post-injection. The two rats with mildmoderate inflammation also had significant reductions (35\% and $55 \%$ of baseline values at 13-14 days postinjection) in CBG levels. Total protein levels in plasma samples did not differ in rats over the course of the study in relation to the severity of inflammation (data not shown).

\section{Plasma CBG and hepatic Cbg mRNA levels in relation to clinical score}

Plasma CBG levels at termination declined in relation to clinical score (main effect of inflammation severity, $P=0.0001$; Fig. 2A). Rats with severe inflammation had significantly lower CBG levels when compared with saline-treated controls or adjuvant-treated rats that did not develop inflammation $(P<0.001)$. Significant differences were also seen in $C b g$ mRNA levels in livers collected on the day of termination (main effect of inflammation severity, $P<0.02$; Fig. $2 \mathrm{~B}$ ). When compared with saline-treated rats or adjuvant-treated rats that did not develop inflammation, post hoc tests revealed that animals with severe inflammation had lower $C b g$ mRNA levels $(P<0.05)$.

Western blotting was used to assess the integrity of CBG in plasma samples taken at termination. An $\sim 5 \mathrm{kDa}$ reduction in the apparent molecular size of CBG was evident in rats with mild-moderate and severe inflammation (Fig. 2C, lanes 4 and 5), consistent with the cleavage of its RCL (Gardill et al. 2012). This was also observed in some adjuvant-treated rats without clinical signs of inflammation (Fig. 2C, lane 3), suggesting the presence of an underlying inflammatory state in those animals. As expected, this evidence of CBG proteolysis coincided with decreased plasma CBG levels as

Published by Bioscientifica Ltd. 
A

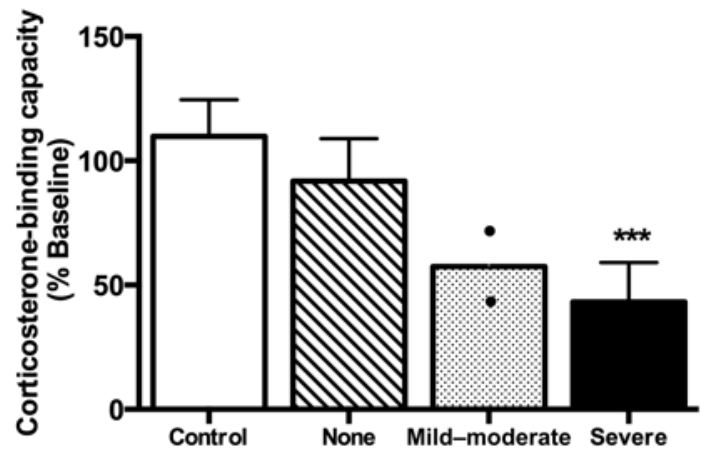

(4)

(15)

(2)

(3)

B

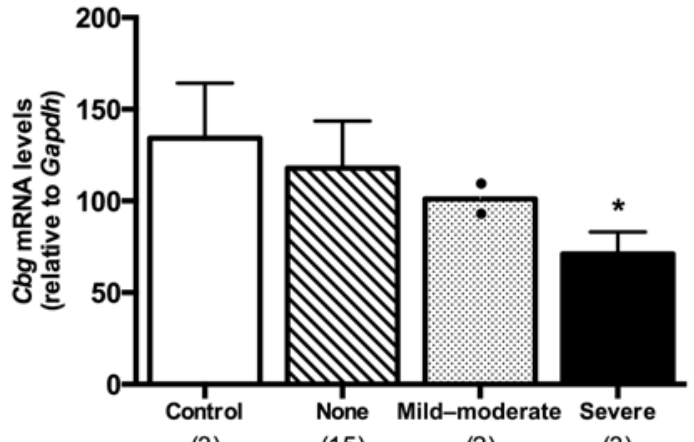

(3)

(15)
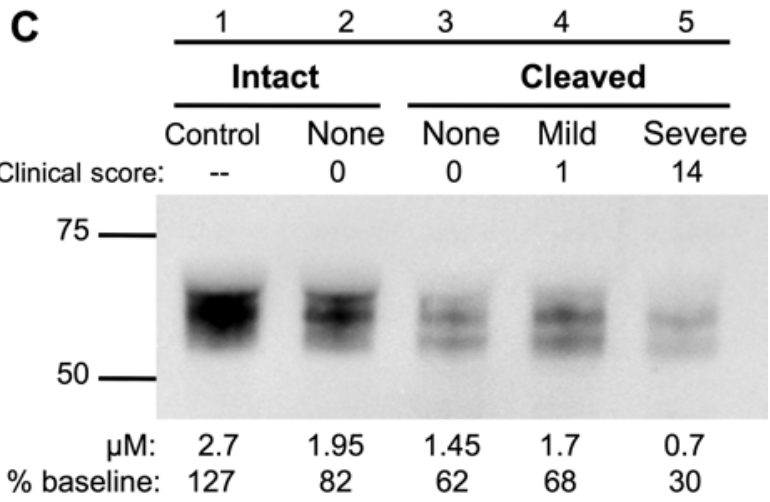

Figure 2

Plasma CBG (A) and liver Cbg mRNA (B) levels, and evidence of CBG proteolysis (C) in relation to clinical score (none, mild-moderate or severe). In $A$ and $B$, the numbers of animals in each group are shown in parentheses, and rats $(n=2)$ with mild-moderate inflammation were not included in statistical analyses. Rats with severe inflammation were compared with both saline-treated control animals and adjuvant-treated animals without clinincal signs of inflammation (none). (A) Plasma CBG levels decreased in relation to clinical score with a significant decrease in rats with severe inflammation (main effect of inflammation severity, $\mathrm{F}$ $(2,19)=14.85, P=0.0001)$. (B) Liver Cbg mRNA levels were significantly lower in animals with severe inflammation (main effect of inflammation severity, $F(2,17)=5.59, P<0.02)$. Data in $A$ and $B$ are presented as mean \pm s.D. Tukey's post hoc: ${ }^{*} P<0.05 ; * * * P<0.001$, (C) Representative Western blotting illustrating the proteolysis of plasma CBG, as indicated by an $\sim 5 \mathrm{kDa}$ size reduction (lanes 3-5), in concert with reductions in CBG corticosteronebinding capacity $(\mu \mathrm{M})$ when compared as a percentage of pre-treatment values (shown below). Note that CBG proteolysis was also observed in some animals before clinical signs of inflammation (none, lane 3).
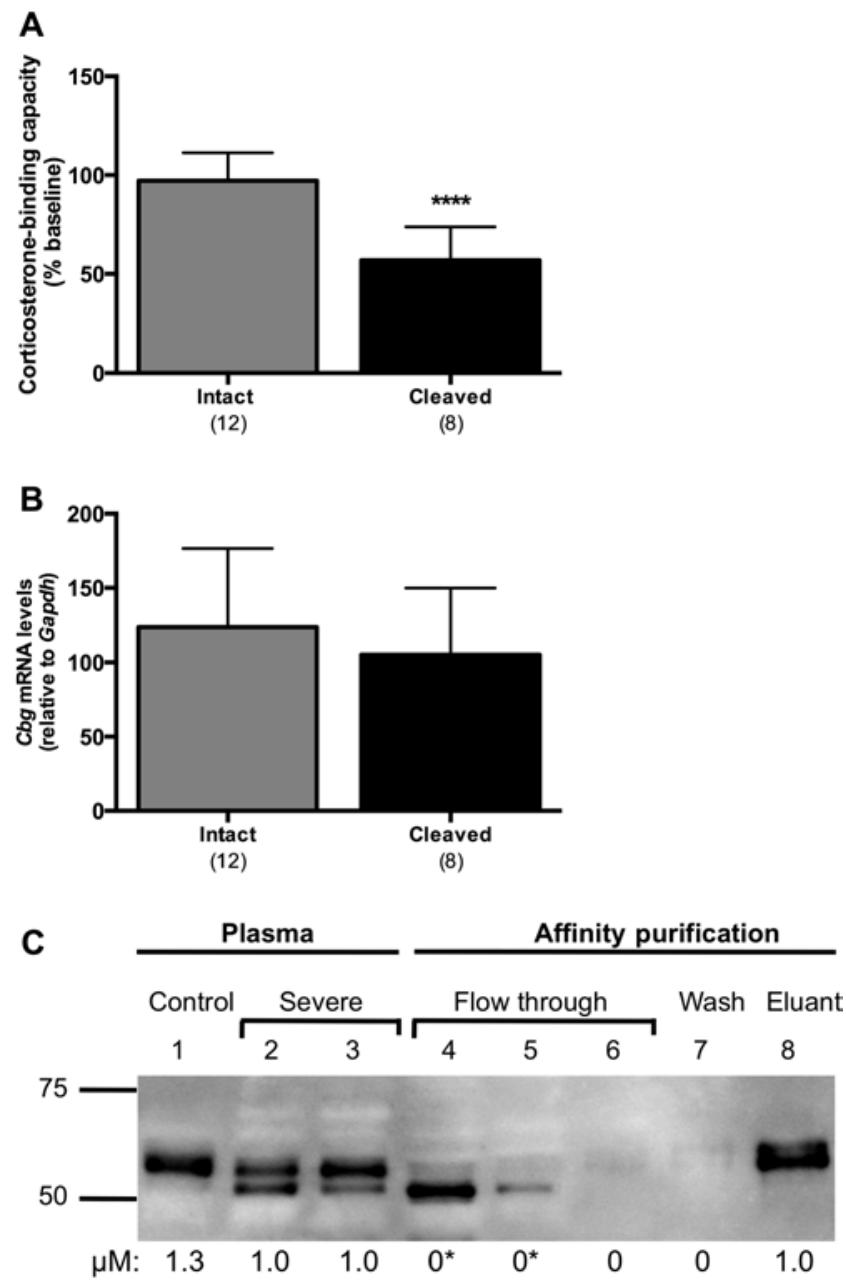

Figure 3

Proteolysis of CBG is associated with reduced plasma CBG levels without changes in liver Cbg mRNA levels, and evidence that cleaved CBG in plasma lacks steroid-binding activity. (A) Rats with cleaved CBG had significantly lower plasma CBG values than rats with intact CBG. (B) Liver Cbg mRNA levels were similar irrespective of CBG proteolysis status. In $A$ and $B$, samples were grouped for analysis based on CBG integrity, as assessed by Western blotting (Fig. 2C), and classified as being either intact or cleaved. The numbers of animals in each group are shown in parentheses, and data are presented as mean \pm S.D. $* * * * P<0.0001$. (C) Plasma from rats with severe inflammation (lanes 2 and 3) were pooled and purified by steroid-affinity chromatography. Cleaved CBG did not bind the steroid-affinity matrix and eluted in the flow through (lanes 4 and 5). Intact CBG bound to the steroid-affinity matrix and was eluted with buffer containing excess corticosterone (lane 8). CBG-corticosteronebinding capacity values $(\mu \mathrm{M})$ are shown under each lane. There was no detectable CBG steroid-binding activity (*) in the flow-through fractions that contained cleaved CBG (lanes 4 and 5). Intact CBG (lane 8) exhibited full steroid-binding activity. An intact control (saline) sample is shown (lane 1) for comparison.

determined in the corticosterone-binding capacity assay (Fig. 2C). Therefore, on the day of termination, decreases in the corticosterone-binding capacity of plasma CBG in animals with severe inflammation are associated

Published by Bioscientifica Ltd. 
with decreased $\mathrm{Cbg}$ mRNA levels, as well as evidence of proteolytic cleavage of CBG.

\section{Plasma CBG and hepatic Cbg mRNA levels in relation to CBG proteolysis}

Plasma samples taken at termination were classified into two groups depending on the integrity of $\mathrm{CBG}$ as assessed by Western blotting: i.e. samples in which CBG proteolysis was either evident (cleaved) or not (intact), as illustrated in Fig. 2C. Reductions in CBG levels were found in rats with evidence of cleaved CBG (Fig. 3A), when compared with rats with intact CBG $(P<0.0001)$. When classifying samples in this way, no differences were found in corresponding liver Cbg mRNA levels (Fig. 3B), suggesting that $\mathrm{CBG}$ proteolysis precedes changes in liver Cbg mRNA levels.

Next, we investigated whether cleaved CBG in plasma samples retained high-affinity corticosteronebinding activity. Plasma samples from rats with severe inflammation (Fig. 3C, lanes 2 and 3) were pooled and CBG was captured by HACA-Sepharose affinity column chromatography. Cleaved CBG, as indicated by an $\sim 5 \mathrm{kDa}$ size reduction, failed to bind the affinity column and eluted in the flow-through fractions (Fig. 3C, lanes 4 and 5). Moreover, the flow-through fractions containing the cleaved CBG had no detectable CBG-corticosterone binding activity. There was no immunoreactive CBG or corticosteronebinding activity in the wash fraction (Fig. 3C, lane 7). Importantly, the CBG eluting from the affinity column using excess $(200 \mu \mathrm{M})$ corticosterone (Fig. 3C, lane 8) appeared to be intact and retained full corticosteronebinding activity.

\section{Plasma cytokine biomarkers of inflammation and spleen weight}

Pro- and anti-inflammatory cytokine levels were measured in plasma samples collected at termination (Fig. 4A, B, C, D, E, F and G). Measurements in samples from salinetreated control animals and adjuvant-treated animals were classified according to whether CBG was intact or cleaved (Fig. 2C). No significant differences were found in plasma levels of IL-5 (Fig. 4A). Trends for a main effect of CBG proteolysis were found for the anti-inflammatory cytokines IL-4 (Fig. 4B, $P=0.051$ ) and IL-10 (Fig. 4C, $P=0.056)$, as well as the pro-inflammatory cytokine IFN- $\gamma$ (Fig. 4D, $P=0.057$ ). Planned pairwise comparisons revealed significantly elevated IL-4, IL-10 and IFN- $\gamma$ levels only in rats with cleaved CBG, when compared with saline-treated controls $(P<0.05)$.

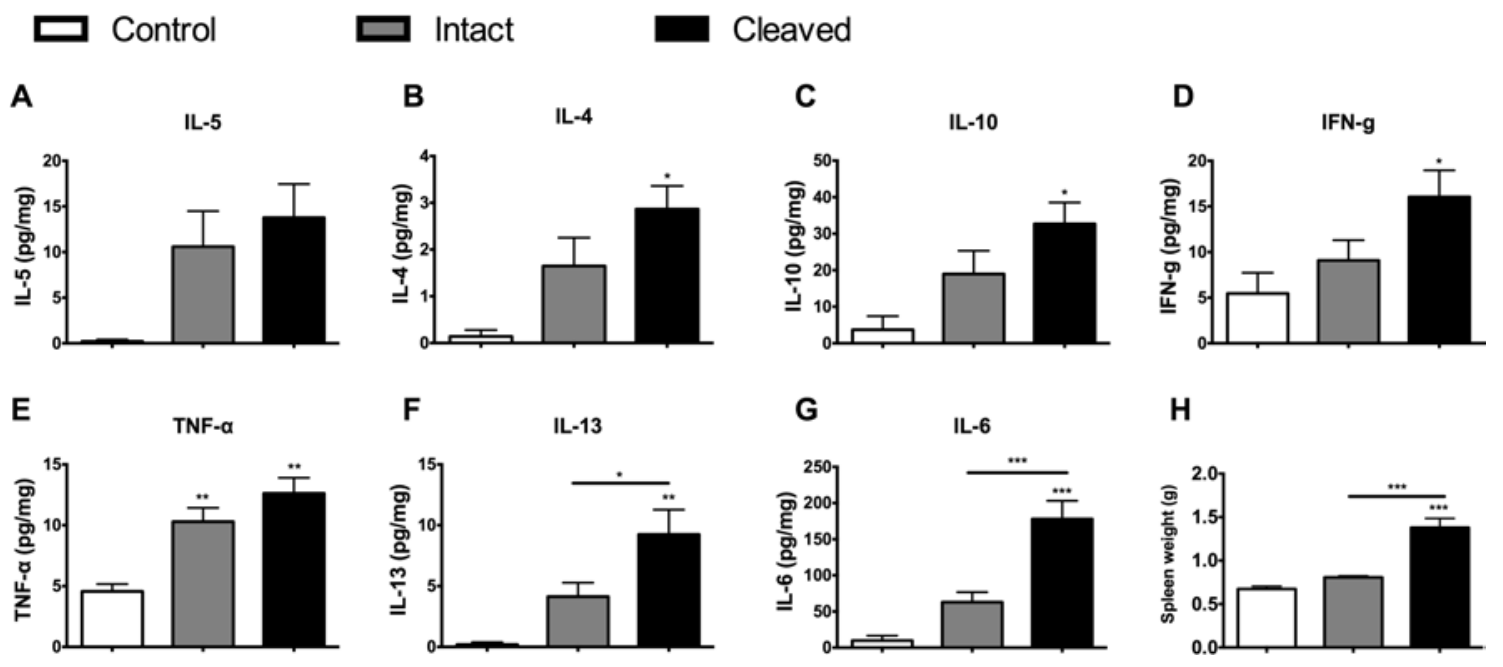

\section{Figure 4}

Associations among CBG proteolysis, plasma cytokine levels ( $A, B, C, D, E, F$ and $G$ ) and spleen weights (H). As in Fig. 3, results from adjuvant-treated rats were grouped based on CBG proteolysis status (i.e. cleaved vs intact CBG). When compared with control (saline-treated) rats, all plasma cytokines, apart from IL-5 (A), were significantly increased in rats with evidence of CBG proteolysis, as was spleen weight (H). TNF- $\alpha(E$, main effect of CBG proteolysis, $F(2,23)=7.30, P<0.004)$ showed a treatment effect with significantly increased levels in adjuvant-treated animals (intact and cleaved $C B G$ ) when compared with controls. Plasma levels of IL-13 ( $F$, main effect of CBG proteolysis, $F(2,23)=6.36, P<0.007)$, IL-6 (G, main effect of CBG proteolysis, $F(2,23)=16.50, P<0.001)$ and spleen weight $(H$, main effect of $C B G$ proteolysis, $F(2,23)=30.47, P<0.001)$ were significantly increased in rats with cleaved CBG vs intact CBG. Cytokine levels were Blom transformed for statistical analysis; untransformed data (pg/mL) are presented as mean \pm s.E.M. Control, $n=4$; Intact, $n=12$; Cleaved, $n=8$. Fischer's post hoc: ${ }^{*} P<0.05 ; * * P<0.01 ; * * * P<0.001$. 

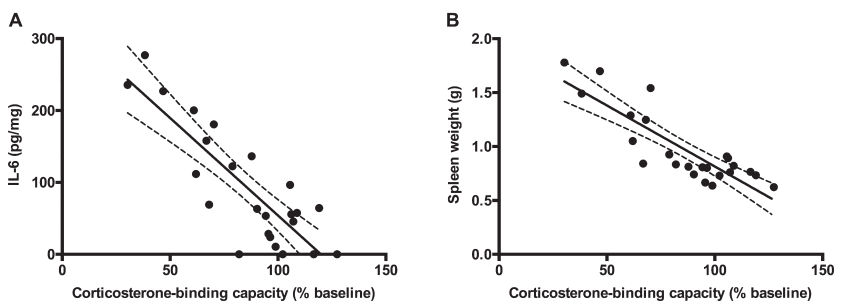

Figure 5

Strong negative correlations between plasma CBG and IL-6 levels and CBG and spleen weights. Data were analysed using linear regression, with plasma CBG levels expressed as a \% of pre-treatment values. (A) Plasma CBG and IL-6 levels $\left(r^{2}=0.71\right)$ and $(B)$ plasma CBG and spleen weights $\left(r^{2}=0.73\right)$, were negatively correlated. The best-fit linear regression line (solid) and $95 \%$ confidence interval (dotted) are also shown.

A significant main effect of $\mathrm{CBG}$ proteolysis was found for TNF- $\alpha(P<0.004)$, IL-13 $(P=0.007)$ and IL-6 $(P<0.001)$, respectively. A treatment effect was found for the pro-inflammatory cytokine TNF- $\alpha$, with post hoc tests revealing that adjuvant-treated animals (both intact and cleaved CBG) had higher cytokine levels compared with control animals (Fig. 4E, $P<0.01$ ). A different pattern was observed for the anti-inflammatory cytokine IL-13 for which post hoc tests indicated that plasma levels were significantly increased in rats with cleaved CBG, when compared with saline-treated controls $(P<0.001)$ or rats with intact CBG $(P<0.05)$, respectively (Fig. $4 \mathrm{~F})$. In terms of changes in plasma cytokine levels, the largest increases were seen for IL-6 (Fig. 4G), with post hoc tests indicating significant increases in rats with cleaved CBG, as compared with the saline-treated controls or rats with intact CBG $(P<0.001)$. Similar to IL-6, spleen weight (Fig. 4H) was very significantly associated with $\mathrm{CBG}$ proteolysis (main effect of CBG proteolysis, $P<0.001$ ). Post hoc tests also indicated that rats with cleaved CBG had significantly elevated spleen weights, when compared with the salinetreated controls or adjuvant-treated rats with intact CBG $(P<0.001)$. Furthermore, there were strong negative correlations between plasma CBG and IL-6 levels (Fig. 5A, $\left.r^{2}=0.71\right)$ and between plasma CBG levels and spleen weights (Fig. $5 \mathrm{~B}, r^{2}=0.73$ ) but not between plasma CBG and IL-13 levels $\left(r^{2}=0.22\right)$.

\section{Discussion}

Changes in plasma CBG levels occur during inflammation in humans, with very low levels reported during sepsis (Ho et al. 2006), septic shock (Pugeat et al. 1989, Ho et al. 2006, Bendel et al. 2008), burn injuries (Bernier et al. 1998) and after open heart surgery (Tinnikov et al. 1996).
In addition, decreased CBG levels have been reported after thermal injuries to mice and rats (Garrel et al. 1993, D'Elia et al. 2005) and in pigs treated with lipopolysaccharide (Carroll et al. 2003). These latter studies model acute, all-or-none inflammation, while the adjuvant-induced inflammation model we have used allows those rats that developed inflammation to be compared with those that did not. In doing so, it was possible to monitor temporal changes in the plasma levels of $\mathrm{CBG}$ and inflammatory markers, as inflammation developed at different rates and degrees of severity.

In adjuvant-treated rats, the corticosterone-binding capacity of plasma CBG decreased $30-50 \%$ at 24 -h postinjection, irrespective of whether or not rats eventually developed inflammation. Based on previous reports (Billiau \& Matthys 2001), we suspect that an initial inflammatory response to the complete Freund's adjuvant is responsible for decreases in plasma CBG levels because this did not occur in saline-treated controls. However, this initial response was short-lived and CBG levels in adjuvant-treated animals returned to baseline by day 3 post-injection. Over the subsequent experimental period, we found consistent decreases in plasma CBG levels of $40-80 \%$ in those animals that developed inflammation, and the magnitude of this decline aligned with the severity of inflammation, as indexed by clinical scores. Notably, marked (50\%) decreases in plasma CBG levels occurred 4 days before any clinical symptoms of inflammation were evident. Such dynamic changes in CBG levels before or during inflammation are expected to modulate the availability of corticosterone to its target cells, thereby affecting the inflammatory reaction as well as the healing process. When considered together, these results suggest that CBG may be a useful biomarker of inflammation onset and severity.

As noted previously (Bodnar et al. 2015), significant decreases in plasma CBG levels occurred in adjuvanttreated rats that developed severe inflammation, and we have now defined the mechanisms responsible for this. At day 14 or 16 post-adjuvant injection (termination), rats with severe inflammation had reduced plasma CBG levels and this was associated with an $\sim 5 \mathrm{kDa}$ reduction in CBG molecular size by Western blotting. Changes in the carbohydrate composition of plasma proteins have been reported to occur during acute inflammation, including decreases in core fucosylation (Rombouts et al. 2016). However, small changes in the composition of the six N-linked oligosaccharides associated with rat CBG will not result in detectable differences in molecular size by Western blotting. It is also known that the complete loss

Published by Bioscientifica Ltd. 
of $\mathrm{N}$-glycosylation of human CBG at Asn238 causes a loss of steroid-binding activity (Avvakumov et al. 1993); however, there is no evidence that compositional changes in the N-linked glycosylation of CBG, in any species, adversely effect its steroid-binding activity. Differences in N-linked oligosaccharide composition will not account for the substantial reduction in the molecular size of CBG, or the complete loss of steroid-binding activity associated with immune-reactive CBG that does not interact with the steroid-affinity chromatography matrix, and we, therefore, conclude that these observations are the result of proteolytic cleavage.

It has been reported that cleaved CBG can be detected in human blood samples using ELISAs with highly specific monoclonal antibodies that discriminated between CBG with an intact vs cleaved RCL (Lewis \& Elder 2011), but direct evidence that RCL cleavage of CBG actually occurred in these samples is lacking. A size reduction in CBG consistent with RCL cleavage has never been observed in human blood samples, and it has been postulated that human CBG is rapidly removed from the blood circulation after RCL cleavage (Mast et al. 1991). In rats, this does not seem to be the case and it appears that cleaved CBG is cleared more slowly from the circulation. However, the site of RCL cleavage and the protease responsible remain to be identified.

The steroid-binding activity of CBG is undetectable in rat plasma samples in which CBG appears to have undergone proteolysis, and this is in line with a marked loss in cortisol-binding affinity observed when the RCL of human CBG is cleaved by neutrophil elastase (Hammond et al. 1990), chymotrypsin (Simard et al. 2015) or the bacterial proteinase, LasB (Simard et al. 2014). However, our observations of in vivo rat CBG proteolysis under pathophysiological conditions contrasts with a previous report that E. coli-produced rat CBG, mutated to allow for cleavage by human neutrophil elastase, only undergoes a two-fold reduction in binding affinity (Gardill et al. 2012). We attribute this discrepancy to the fact that E. coliexpressed CBG is not glycosylated and has about a 10-fold lower affinity for corticosterone when compared with native CBG in rat blood samples (Gardill et al. 2012). It is known that the $\mathrm{N}$-glycosylation of human CBG is critically important for the formation of a high-affinity binding site (Avvakumov et al. 1993, Avvakumov \& Hammond 1994), and the RCLs of human and rat CBGs both contain $\mathrm{N}$-glycosylation sites, but in different locations (Hammond et al. 1991). It is possible that $\mathrm{N}$-glycosylation within the RCL of rat CBG influences how, and in what location, it is cleaved by a protease that allows for RCL insertion and the subsequent protein conformational rearrangement that disrupts the high-affinity steroid-binding properties of CBG (Lin et al. 2010). Unexpectedly, plasma CBG proteolysis was also evident in rats that developed mild-moderate inflammation, as well as in a subset of rats that did not display clinical signs of inflammation. However, significant reductions in plasma CBG were evident in rats where $\mathrm{CBG}$ proteolysis appears to have occurred, despite the fact that this did not coincide with a reduction in liver $\mathrm{Cbg}$ mRNA levels, which suggests that plasma CBG proteolysis occurs before changes in liver Cbg mRNA levels.

Plasma CBG production by the liver is downregulated by glucocorticoids (Smith \& Hammond 1992) and cytokines (Emptoz-Bonneton et al. 1997). Although plasma corticosterone levels were not measured in this study, they were increased in our previous study using the same experimental protocol, with the highest levels found in animals with severe inflammation (Bodnar et al. 2015). In this model, increased levels of IL-6, as seen here, and corticosterone, as seen previously (Bodnar et al. 2015), are likely contributors to the significant decreases in liver Cbg mRNA levels that we have now observed in rats with severe inflammation. This is not entirely surprising given the presence of a cis-regulatory element for IL-6 in the rat Cbg promoter (Underhill \& Hammond 1995), and the established role of IL-6 in the acute phase response during inflammation (Fonseca et al. 2009). This is also in line with inverse relationships between IL- 6 and plasma CBG levels in humans (Bernier et al. 1998, Tsigos et al. 1998), and studies in human hepatoblastoma-derived (HepG2) cells, where an IL-6-induced reduction in CBG production (Emptoz-Bonneton et al. 1997) was associated with decreased Cbg mRNA stability (Bartalena et al. 1993). Moreover, the strong negative correlation we observed between plasma CBG and IL-6 levels further supports the proposition that IL- 6 inhibits CBG production during inflammation. Together, our results suggest that the mechanisms responsible for decreases in plasma CBG levels during inflammation are multi-factorial and occur in a sequential manner. First, the RCL of CBG undergoes proteolysis rendering it essentially non-functional as a steroid-binding protein, thereby amplifying free plasma corticosterone levels. Further decreases to plasma CBG levels are then caused by decreases in liver Cbg mRNA levels, mediated by increased IL- 6 and corticosterone levels.

Activated immune cells produce a wide variety of cytokines, and cytokine levels increase in the circulation during inflammation (Choy \& Panayi 2001,

Published by Bioscientifica Ltd 
Ramadori \& Armbrust 2001). We have now found that animals that showed evidence of CBG proteolysis also had increased cytokine (IFN- $\gamma$, TFN $\alpha$, IL-4, IL-6, IL-10 and IL-13) levels, in association with large increases in spleen weight, which is a recognized marker of inflammation. These changes in plasma cytokine levels are expected to act together with increases in corticosterone levels and bioavailability to repress the production of cytokines in an attempt to alleviate cytokine-mediated tissue damage in rats with cleaved CBG. Increases in the plasma levels of positive acute phase plasma proteins, including other SERPINA family members, such as alpha- 1 antitrypsin (SERPINA1) that inhibits the activities of neutrophil elastase in humans, may have occurred in animals that developed inflammation. Although this may afford some protection to $\mathrm{CBG}$ proteolysis, there is little information about the roles of alpha-1 antitrypsin and other related SERPINAs, or their target proteases, in rats in relation to inflammation.

The fact that both plasma IL- 6 and IL-13 levels are markedly elevated in animals with cleaved CBG, while only IL-6 levels were inversely correlated with plasma CBG levels, suggests that these cytokines function in different ways. In contrast to IL-6, the lack of any relationship between plasma CBG and IL-13 levels suggests that IL-13 does not influence CBG production or proteolysis. Moreover, the coincidence of increased cytokine levels and spleen weights in rats with cleaved CBG indicates an underlying inflammatory response, even in the absence of clinical symptoms. Although some rats with cleaved CBG did not display overt signs of inflammation over the experimental period, it is possible that these may have developed if the study had been extended. Nevertheless, these data indicate that evidence of CBG proteolysis in plasma samples is a potential pre-symptomatic biomarker of inflammation.

In conclusion, our time course study of CBG changes during inflammation demonstrates that significant decreases in the corticosterone-binding capacity of plasma CBG occur in rats that developed inflammation, with the magnitude of the decline matching the clinical severity. Notably, large decreases in plasma CBG levels occur before clinical signs of inflammation. At termination, significant decreases in the corticosterone-binding capacity of CBG appear to coincide with CBG proteolysis that causes a loss of its steroid-binding activity. Significant increases in pro- and anti-inflammatory plasma cytokine levels, as well as increased spleen weights, were all associated with evidence of CBG proteolysis. These novel findings suggest that CBG proteolysis is a marker of active inflammation and, perhaps even more importantly, is a prognostic indicator of inflammation onset. In addition, the fact that rats with evidence of plasma CBG proteolysis had similar liver $\mathrm{Cbg}$ mRNA levels to rats with intact CBG suggests that cleavage of plasma CBG occurs before any reductions in hepatic CBG production occur. Overall, our data suggest that changes in $\mathrm{CBG}$, particularly $\mathrm{CBG}$ proteolysis, are an early, pre-symptomatic marker of inflammation and a useful biomarker of inflammation onset and severity.

\section{Declaration of interest}

The authors have declared that they have no conflict of interest that could be perceived as prejudicing the impartiality of the research reported.

\section{Funding}

This work was supported by: the Canadian Institutes of Health Research (Operating Grant MOP-111102) to G L H; NIH/NIAAA (RO1 AA022460 and R37 AA007789) and NeuroDevNet (Canadian Networks of Centres of Excellence) to J W; and the Natural Sciences and Engineering Research Council of Canada (CGS-D award) to T S B. G L H holds a Tier 1 Canada Research Chair in Reproductive Health.

\section{Author contribution statement}

L A H and T S B carried out the experiments and analysed the data. L A H and G L H wrote the manuscript. All authors designed the study, had final approval of the submitted version of the manuscript and have read and agreed with the manuscript written.

\section{Acknowledgements}

The authors wish to thank Dr Timothy Kieffer for generously providing access to the QuickPlex, and Travis Webber for his assistance. We also thank Wayne Yu and Linda Ellis for their assistance with animal procedures.

\section{References}

Avvakumov GV \& Hammond GL 1994 Glycosylation of human corticosteroid-binding globulin. Differential processing and significance of carbohydrate chains at individual sites. Biochemistry 33 5759-5765. (doi:10.1021/bi00185a012)

Avvakumov GV, Warmels-Rodenhiser S \& Hammond GL 1993 Glycosylation of human corticosteroid-binding globulin at aspargine 238 is necessary for steroid binding. Journal of Biological Chemistry 268 862-866.

Bartalena L, Hammond GL, Farsetti A, Flink IL \& Robbins J 1993 Interleukin- 6 inhibits corticosteroid-binding globulin synthesis by human hepatoblastoma-derived (hep G2) cells. Endocrinology 133 291-296. (doi:10.1210/endo.133.1.8391424)

Bendel S, Karlsson S, Pettila V, Loisa P, Varpula M, Ruokonen E \& Finnsepsis Study Group 2008 Free cortisol in sepsis and septic shock. Anesthesia and Analgesia 106 1813-1819. (doi:10.1213/ ane.0b013e318172fdba) 
Bernier J, Jobin N, Emptoz-Bonneton A, Pugeat MM \& Garrel DR 1998 Decreased corticosteroid-binding globulin in burn patients: relationship with interleukin-6 and fat in nutritional support. Critical Care Medicine 26 452-460. (doi:10.1097/00003246199803000-00014)

Billiau A \& Matthys P 2001 Modes of action of freund's adjuvants in experimental models of autoimmune diseases. Journal of Leukocyte Biology 70 849-860.

Bodnar TS, Hill LA, Taves MD, Yu W, Soma KK, Hammond GL \& Weinberg J 2015 Colony-specific differences in endocrine and immune responses to an inflammatory challenge in female sprague dawley rats. Endocrinology 156 4604-4617. (doi:10.1210/en.20151497)

Brattsand R \& Linden M 1996 Cytokine modulation by glucocorticoids: mechanisms and actions in cellular studies. Alimentary Pharmacology \& Therapeutics 10 (Supplement 2) 81-90. (doi:10.1046/j.13652036.1996.22164025.x)

Carroll JA, Gaines AM, Spencer JD, Allee GL, Kattesh HG, Roberts MP \& Zannelli ME 2003 Effect of menhaden fish oil supplementation and lipopolysaccharide exposure on nursery pigs. I. Effects on the immune axis when fed diets containing spray-dried plasma. Domestic Animal Endocrinology 24 341-351. (doi:10.1016/S0739. 7240(03)00017-1)

Choy EH \& Panayi GS 2001 Cytokine pathways and joint inflammation in rheumatoid arthritis. New England Journal of Medicine 344 907-916. (doi:10.1056/NEJM200103223441207)

D'Elia M, Patenaude J, Hamelin C, Garrel DR \& Bernier J 2005 Corticosterone binding globulin regulation and thymus changes after thermal injury in mice. American Journal of Physiology: Endocrinology and Metabolism 288 E852-E860. (doi:10.1152/ajpendo.00407.2004)

Emptoz-Bonneton A, Crave JC, LeJeune H, Brebant C \& Pugeat M 1997 Corticosteroid-binding globulin synthesis regulation by cytokines and glucocorticoids in human hepatoblastoma-derived (HepG2) cells. Journal of Clinical Endocrinology and Metabolism 82 3758-3762. (doi:10.1210/jcem.82.11.4362)

Fonseca JE, Santos MJ, Canhao H \& Choy E 2009 Interleukin-6 as a key player in systemic inflammation and joint destruction. Autoimmunity Reviews 8 538-542. (doi:10.1016/j.autrev.2009.01.012)

Gagliardi L, Ho JT \& Torpy DJ 2010 Corticosteroid-binding globulin: the clinical significance of altered levels and heritable mutations. Molecular and Cellular Endocrinology 316 24-34. (doi:10.1016/j. mce.2009.07.015)

Gardill BR, Vogl MR, Lin HY, Hammond GL \& Muller YA 2012 Corticosteroid-binding globulin: structure-function implications from species differences. PLOS ONE 7 e52759. (doi:10.1371/journal. pone.0052759)

Garrel DR, Zhang L, Zhao XF \& Hammond GL 1993 Effect of burn injury on corticosteroid-binding globulin levels in plasma and wound fluid. Wound Repair and Regeneration 1 10-14. (doi:10.1046/j.1524475X.1993.10105.X)

Hammond GL 1990 Molecular properties of corticosteroid binding globulin and the sex-steroid binding proteins. Endocrine Reviews $\mathbf{1 1}$ 65-79. (doi:10.1210/edrv-11-1-65)

Hammond GL \& Lahteenmaki PL 1983 A versatile method for the determination of serum cortisol binding globulin and sex hormone binding globulin binding capacities. Clinica Chimica Acta 132 101-110. (doi:10.1016/0009-8981(83)90237-1)

Hammond GL, Smith CL, Paterson NA \& Sibbald WJ 1990 A role for corticosteroid-binding globulin in delivery of cortisol to activated neutrophils. Journal of Clinical Endocrinology and Metabolism 71 34-39. (doi:10.1210/jcem-71-1-34)

Hammond GL, Smith CL \& Underhill DA 1991 Molecular studies of corticosteroid binding globulin structure, biosynthesis and function. Journal of Steroid Biochemistry and Molecular Biology 40 755-762. (doi:10.1016/0960-0760(91)90300-T)
Ho JT, Al-Musalhi H, Chapman MJ, Quach T, Thomas PD, Bagley CJ, Lewis JG \& Torpy DJ 2006 Septic shock and sepsis: a comparison of total and free plasma cortisol levels. Journal of Clinical Endocrinology and Metabolism 91 105-114. (doi:10.1210/jc.2005-0265)

Lewis JG \& Elder PA 2011 Corticosteroid-binding globulin reactive centre loop antibodies recognise only the intact natured protein: elastase cleaved and uncleaved CBG may coexist in circulation. Journal of Steroid Biochemistry and Molecular Biology 127 289-294. (doi:10.1016/j. jsbmb.2011.08.006)

Lewis JG \& Elder PA 2014 The reactive centre loop of corticosteroidbinding globulin (CBG) is a protease target for cortisol release. Molecular and Cellular Endocrinology 384 96-101. (doi:10.1016/j. mce.2014.01.005)

Libert C, Wielockx B, Hammond GL, Brouckaert P, Fiers W \& Elliott RW 1999 Identification of a locus on distal mouse chromosome 12 that controls resistance to tumor necrosis factor-induced lethal shock. Genomics 55 284-289. (doi:10.1006/geno.1998.5677)

Lin HY, Muller YA \& Hammond GL 2010 Molecular and structural basis of steroid hormone binding and release from corticosteroidbinding globulin. Molecular and Cellular Endocrinology 316 3-12. (doi:10.1016/j.mce.2009.06.015)

Mast AE, Enghild JJ, Pizzo SV \& Salvesen G 1991 Analysis of the plasma elimination kinetics and conformational stabilities of native, proteinase-complexed, and reactive site cleaved serpins: comparison of alpha 1-proteinase inhibitor, alpha 1-antichymotrypsin, antithrombin III, alpha 2-antiplasmin, angiotensinogen, and ovalbumin. Biochemistry 30 1723-1730. (doi:10.1021/bi00220a039)

Perogamvros I, Ray DW \& Trainer PJ 2012 Regulation of cortisol bioavailability - effects on hormone measurement and action. Nature Reviews. Endocrinology 8 717-727. (doi:10.1038/nrendo.2012.134)

Petersen HH, Andreassen TK, Breiderhoff T, Brasen JH, Schulz H, Gross V, Grone HJ, Nykjaer A \& Willnow TE 2006 Hyporesponsiveness to glucocorticoids in mice genetically deficient for the corticosteroid binding globulin. Molecular and Cellular Biology 26 7236-7245. (doi:10.1128/MCB.00400-06)

Pugeat M, Bonneton A, Perrot D, Rocle-Nicolas B, Lejeune H, Grenot C, Dechaud H, Brebant C, Motin J \& Cuilleron CY 1989 Decreased immunoreactivity and binding activity of corticosteroid-binding globulin in serum in septic shock. Clinical Chemistry 35 1675-1679.

Ramadori G \& Armbrust T 2001 Cytokines in the liver. European Journal of Gastroenterology \& Hepatology 13 777-784. (doi:10.1097/00042737200107000-00004)

Rombouts Y, Jonasdottis HS, Hipgrave Ederveen AL, Reidine KR, Jansen BC, Freysdottir J, Hardardottir I, Ioan-Facsinay A, Giera M \& Wuhrer M 2016 Acute phase inflammation is characterized by rapid changes in plasma/peritoneal fluid N-glycosylation in mice. Glycoconjugate Journal 33 457-470. (doi:10.1007/s10719-015-9648-9)

Sapolsky RM, Romero LM \& Munck AU 2000 How do glucocorticoids influence stress responses? integrating permissive, suppressive, stimulatory, and preparative actions. Endocrine Reviews 21 55-89. (doi:10.1210/edrv.21.1.0389)

Scrocchi LA, Hearn SA, Han VK \& Hammond GL 1993 Corticosteroidbinding globulin biosynthesis in the mouse liver and kidney during postnatal development. Endocrinology 132 910-916. (doi:10.1210/ endo.132.2.8425503)

Seralini GE, Underhill CM, Smith CL, Nguyen VT \& Hammond GL 1989 Biological half-life and transfer of maternal corticosteroid-binding globulin to amniotic fluid in the rabbit. Endocrinology 125 1321-1325. (doi:10.1210/endo-125-3-1321)

Simard M, Hill LA, Underhill CM, Keller BO, Villanueva I, Hancock RE \& Hammond GL 2014 Pseudomonas aeruginosa elastase disrupts the cortisol-binding activity of corticosteroid-binding globulin. Endocrinology 155 2900-2908. (doi:10.1210/en.2014-1055)

Simard M, Hill LA, Lewis JG \& Hammond GL 2015 Naturally occurring mutations of human corticosteroid-binding globulin. Journal of http://joe.endocrinology-journals.org

DOI: $10.1530 / \mathrm{JOE}-16-0047$
() 2016 Society for Endocrinology Printed in Great Britain 
Clinical Endocrinology and Metabolism 100 E129-E139. (doi:10.1210/ jc.2014-3130)

Simon HU 2003 Neutrophil apoptosis pathways and their modifications in inflammation. Immunological Reviews 193 101-110. (doi:10.1034/ j.1600-065X.2003.00038.x)

Smith CL \& Hammond GL 1992 Hormonal regulation of corticosteroidbinding globulin biosynthesis in the male rat. Endocrinology 130 2245-2251. (doi:10.1210/endo.130.4.1547738)

Tinnikov AA, Legan MV, Sheveluk NA, Cvetovskaya GA, Naumenko SE \& Sidelnikov SG 1996 Corticosteroid and immune responses to cardiac surgery. Steroids 61 411-415. (doi:10.1016/0039-128X(96)00060-8)

Tsigos C, Kyrou I, Chrousos GP \& Papanicolaou DA 1998 Prolonged suppression of corticosteroid-binding globulin by recombinant human interleukin-6 in man. Journal of Clinical Endocrinology and Metabolism 83 3379. (doi:10.1210/jcem.83.9.5100-5)

Turnbull AV \& Rivier CL 1999 Sprague-dawley rats obtained from different vendors exhibit distinct adrenocorticotropin responses to inflammatory stimuli. Neuroendocrinology 70 186-195. (doi:10.1159/000054475)

Underhill DA \& Hammond GL 1995 Cis-regulatory elements within the proximal promoter of the rat gene encoding corticosteroid-binding globulin. Gene 162 205-211. (doi:10.1016/0378-1119(95)00337-6)

Zhang X, Lan N, Bach P, Nordstokke D, Yu W, Ellis L, Meadows GG \& Weinberg J 2012 Prenatal alcohol exposure alters the course and severity of adjuvant-induced arthritis in female rats. Brain, Behavior, and Immunity 26 439-450. (doi:10.1016/j.bbi.2011.11.005)

Received in final form 26 May 2016

Accepted 3 June 2016 (c) 2016 Society for Endocrinology Printed in Great Britain
Published by Bioscientifica Ltd 